\title{
CAN SECOND EYE CATARACT EXTRACTION BE JUSTIFIED?
}

\author{
ALISTAIR LAIDLAW and RICHARD HARRAD \\ Bristol
}

\begin{abstract}
SUMMARY
A prospective study was carried out to test the null hypothesis that there is no additional benefit to be gained from second eye cataract extraction. Twenty-nine patients with unilateral cataract but contralateral pseudophakia completed a questionnaire enquiring into their visual disability and underwent testing of monocular and binocular visual acuity, contrast sensitivity, glare disability, fusion and stereopsis. These tests were repeated 4 months after second eye cataract extraction and lens implantation. Patients universally considered their vision to have been improved by second eye surgery and the prevalence of all symptoms were significantly reduced by this procedure. Normal binocular summation and stereopsis were restored following second eye cataract extraction. Symptomatic patients may benefit from second eye cataract extraction with lens implantation.
\end{abstract}

Cataract extraction with lens implantation is recognised as a cost-effective treatment for severe visual loss due to cataract. ${ }^{1.2}$ Despite an annual expenditure of approximately $£ 100$ million in the United Kingdom, long waiting lists for cataract surgery are common. ${ }^{3}$ A third of this money is spent on 'second eye' operations. ${ }^{4}$ The outcome of cataract extraction has mainly been documented in terms of improved monocular visual function, ${ }^{5}$ and there are few data on the symptomatic disabilities or binocular visual function of patients with good uniocular pseudophakic vision but contralateral cataract. Furthermore there are no data on the benefits, if any, to these patients from undergoing second eye surgery. As a result of the demand for cataract surgery and these limitations in the available outcome data on this procedure there is no consensus as to which patients, if any, should be offered second eye cataract extraction.

Information regarding the need for, and outcome of, second eye surgery is required by ophthalmologists, purchasers and health care planners alike in order to assist with waiting list management and allocation of current

From: University Department of Ophthalmology, Bristol Eye Hospital, Bristol, UK.

Correspondence to: Mr. D. A. H. Laidlaw, Bristol Eye Hospital, Lower Maudlin Street, Bristol BS1 2LX, UK. resources to second eye surgery, and to predict future requirements for ophthalmic surgical services.

It has been widely assumed that the overall visual function of patients with uniocular cataract is as good as that in their better eye. Such patients, however, often complain that their vision is improved if they close their cataractous eye. ${ }^{6}$ When there is equal vision in each eye binocular visual acuity and binocular contrast sensitivity are better than those of either eye tested separately. This phenomenon is known as binocular summation. When the vision in one eye is impaired, binocular visual acuity and contrast sensitivity have been shown to be up to $20 \%$ worse than the values recorded from the better eye alone. This phenomenon is known as binocular inhibition and has been demonstrated in subjects with a uniocular neutral density filter, uniocular glare, uniocular defocus and in patients with very early unilateral cataract. ${ }^{711}$ The importance of this finding in patients with more advanced unilateral cataract requires further investigation as its presence may be considered an important indication for second eye surgery.

We have performed a prospective observational study to test the null hypothesis that there is no additional benefit to be gained from second eye cataract extraction in patients who have already undergone a successful unilateral cataract extraction and lens implantation procedure. To this end we have documented the symptomatic visual disability and both monocular and binocular visual function of 29 patients with unilaterally good vision in one pseudophakic eye and contralateral cataract, before and at least 4 months after second eye surgery.

\section{METHODS}

Patients with a unilateral cataract who had previously undergone uncomplicated contralateral cataract extraction with posterior chamber lens implantation were recruited from the surgical waiting list at Bristol Eye Hospital. Informed consent was obtained, after full explanation of the nature of the procedures to be performed, from all the patients recruited to this study.

The inclusion criteria used in recruiting these patients were: 
1. Unilateral pseudophakia and contralateral cataract.

2. Corrected or uncorrected pseudophakic visual acuity of $6 / 9$ or better.

3. Corrected visual acuity of $6 / 18$ or worse in the cataractous eye.

4. No other identified disease liable to reduce visual acuity, contrast sensitivity or visual field.

5. No previous ocular history of amblyopia or strabismus.

\section{Examination}

All patients were examined by a single examiner (D.A.H.L.). Patients were examined twice: initially before undergoing second eye cataract surgery and subsequently at least 4 months following that procedure. On both occasions they were asked whether they encountered any visual problems when performing their normal activities. These were recorded as spontaneously volunteered symptoms. A standardised series of questions was then presented regarding the following symptoms: glare; having to close one eye to improve vision (binocular inhibition); poor colour perception; blurred vision; poor depth perception; double vision (monocular or binocular); difficulty with the following: seeing to one side (field loss), reading, work or hobbies, mobility and, where relevant, driving difficulties. At the second examination patients were also asked whether their vision had improved as a result of the second cataract extraction and lens implantation procedure.

Patients underwent assessment of (1) monocular and binocular LogMAR Visual acuity (LogMAR Va) from a distance of $4 \mathrm{~m}$, (2) monocular and binocular Pelli-Robson Contrast Sensitivity Threshold (PRCST) tested from a distance of $1 \mathrm{~m}$, (3) monocular PRCST in the presence of a glare light, (4) TNO stereo acuity, and (5) binocular single vision using Worth's four-lights test. Patients' distance spectacle correction (when worn) was measured and the spherical equivalent anisometropia calculated. From this the resulting aniseikonia was calculated. ${ }^{12}$ By subtracting PRCST in the presence of glare from the PRCST without glare the monocular glare disability was calculated. ${ }^{13}$ All tests were performed under recommended lighting conditions.

Patients were examined on a slit lamp biomicroscope to assess the clarity of their pseudophakic posterior capsules and the degree of cataract present. Posterior capsules were graded as: absent, present but optically clear, mildly opacified or opacified. Posterior capsules were graded as opacified if they were thought to reduce visual function sufficiently to warrant a capsulotomy.

When comparing single acuity measurements a 0.16 LogMAR (eight letters) difference is required for a difference to be considered significant. ${ }^{14}$ The Pelli-Robson Contrast Sensitivity chart has letters of equal size arranged in sixteen triplets. Each triplet contains letters of the same contrast, the contrast decreasing by $0.15 \log$ units $(30 \%)$ between each successive triplet. When used at $1 \mathrm{~m}$ Pelli $e t$ $a l .{ }^{15}$ suggest that it measures contrast sensitivity threshold between 0.5 and 2 cycles per degree. Single measurement test-retest reliability data indicate that significance is only achieved when at least a $0.3 \log$ unit (two triplets) increase or decrease in contrast sensitivity threshold is observed. ${ }^{16}$

Glare disability is the elevation of contrast sensitivity threshold occurring in the presence of bright light. ${ }^{13}$ There is no standard means of inducing glare for test purposes. ${ }^{17,18} \mathrm{We}$ induced glare disability using a mainspowered Keeler All Pupil Binocular Indirect Ophthalmoscope (BIO) held $40 \mathrm{~cm}$ from the eye and at an angle of $30^{\circ}$ to the visual axis. The bulb luminance of this instrument was $5000 \mathrm{~cd} / \mathrm{m}^{2}$. The amount of glare induced by a point source depends on its luminance and the angle it subtends from the visual axis. ${ }^{19}$ We have not found reproducible results for binocular glare disability induced with the $\mathrm{BIO}$, perhaps due to the differences in the angle subtended by the light source to each eye and differences in the distance of the light source from each eye.

\section{Statistical Methods}

The results of monocular and binocular LogMAR Va and PRCST conformed to a normal distribution. Parametric statistical analysis was therefore possible. Correction was made for the lack of independence of variation when comparing results derived both from fellow eyes and from monocular and binocular tests. Comparisons of the proportion of patients reporting a visual symptom were performed using chi-squared tests and, where cells contained fewer than five observations, Fisher's exact test. Where proportions are expressed as a percentage the result was rounded up or down to the nearest whole integer.

\section{RESULTS}

Twenty-nine patients aged $57-89$ years (mean 70.9 years) fulfilled the entry and follow-up criteria for this study. They were examined between 4 and 72 months (mean 19.4 months) after their first cataract extraction and were reviewed between 4 and 18 months (mean 9.1 months) after second eye surgery. Twenty-eight patients had an uncomplicated second eye extracapsular extraction with insertion of a posterior chamber lens. Surgery was complicated in one patient by vitreous loss, following which primary anterior chamber lens implantation was performed. This individual's post-operative recovery was uneventful. Three patients had undergone a laser posterior capsulotomy in their second eye prior to being re-examined. These procedures were performed between 6 and 9 months post-operatively. One of these patients developed cystoid macular oedema and at re-examination 12 months after second eye surgery had a acuity of 0.58 LogMAR $(6 / 22)$ in the affected eye. None of the patients were judged to have posterior capsular thickening sufficient to warrant capsulotomy at the time of their second examination.

\section{Symptoms}

Patients with uniocular cataract had symptomatically poor vision. When re-examined these patients unanimously 
considered their vision to have been improved by second eye cataract extraction. Patients volunteered on average 1.6 symptoms (range 1-4) prior to surgery. No symptoms were volunteered post-operatively (Fig. 1).

The average number of symptoms elicited from the ten standard questions pre-operatively was 4.6 (range 2-8). Post-operatively this was reduced to 1.6 (range 1-3). Fig. 2 shows the proportion of patients from whom each symptom was elicited before and after second eye cataract extraction. Each elicited symptom was significantly less prevalent following second eye surgery $(p<0.01)$.

\section{Binocular Single Vision}

Using Worth's four-lights test prior to second eye surgery, suppression of the cataractous eye was demonstrated in one patient. Post-operatively all patients demonstrated binocular single vision.

\section{Stereopsis}

Prior to surgery TNO stereopsis was unrecordable in 12 patients $(41 \%)$ and could be demonstrated to be better than 960 seconds of arc in only 2 patients. Following second eye cataract extraction stereopsis was improved in all patients and $24 / 29(83 \%)$ demonstrated a stereo acuity of 120 seconds of arc or better (Fig. 3). No significant correlation could be demonstrated between pre- and post-operative stereo acuity. Eight of 12 patients shown to have undetectable TNO stereo acuity pre-operatively were able to demonstrate a stereo acuity of 120 seconds of arc or better following second eye surgery. The patient in whom suppression was demonstrated with Worth's four-lights test pre-operatively attained a stereo acuity of 240 seconds of arc post-operatively.

\section{Anisometropia and Aniseikonia}

The mean spherical anisometropia pre-operatively was 1.04 dioptres (range $0-4$ dioptres). Following second eye surgery the mean had decreased to 0.66 dioptres (range $0-4.5$ dioptres). The resulting post-operative mean aniseikonia was $1.6 \%$ (range $0-7 \%$ ). The formula used when performing this calculation assumes a refractive rather than axial anisometropia and a back vertex distance of $12 \mathrm{~mm} .{ }^{12}$ No significant correlation could be demonstrated between patients' post-operative aniseikonia and their TNO stereo acuity $(r=-0.176)$.

\section{Visual Acuity}

The mean pre-operative pseudophakic visual acuity was 0.07 LogMAR (Snellen equivalent 6/7.0). This lies within the normal range quoted by Elliot et al. ${ }^{16}$ for similarly aged normal subjects and can therefore be considered to be a normal visual acuity in patients of this age. The mean visual acuity of the cataractous eye was 0.99 LogMAR $(6 / 57)$.

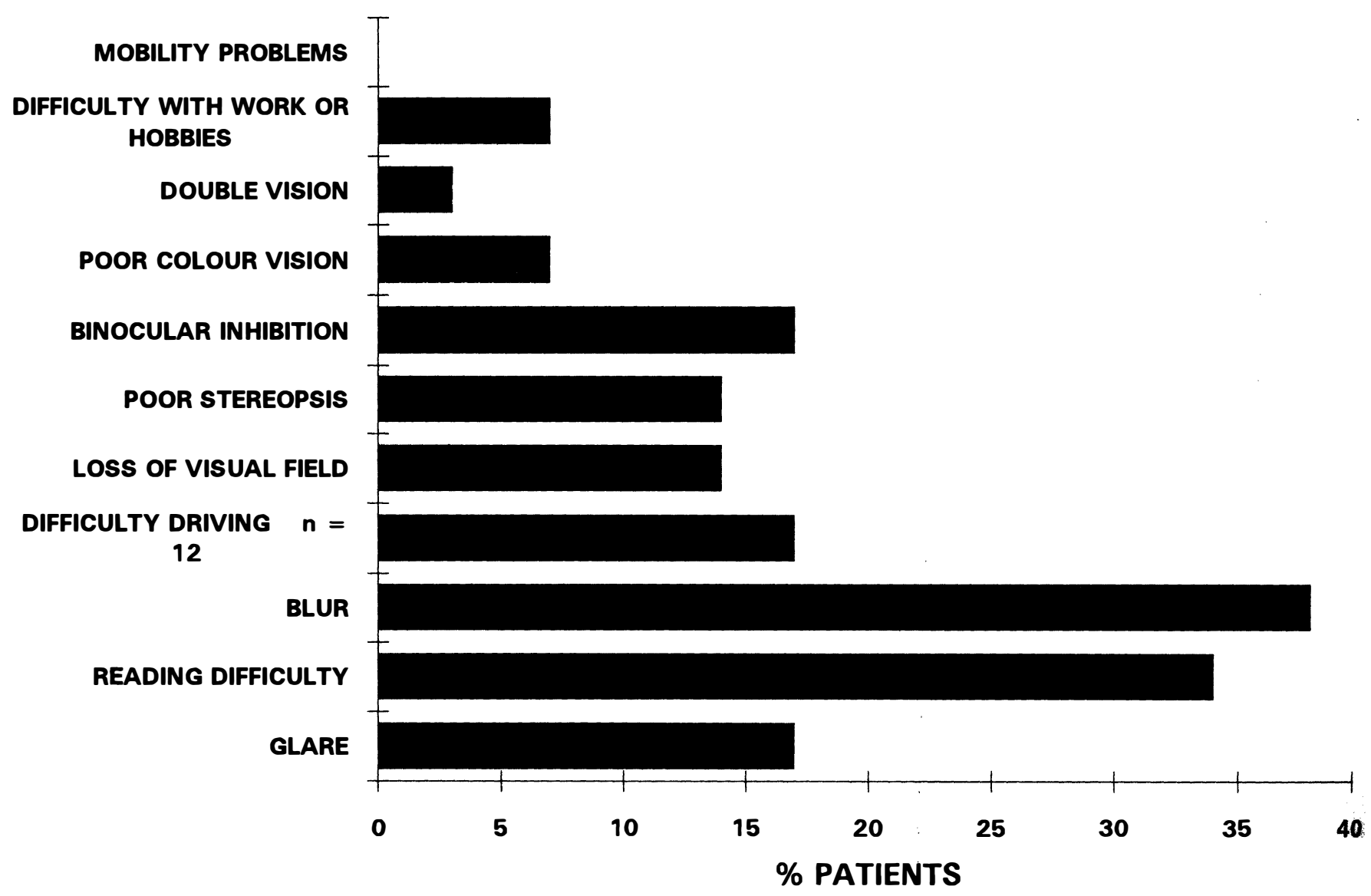

Fig. 1. Percentage of patients volunteering each visual symptom prior to second eye cataract extraction. Following second eye surgery no symptoms were spontaneously volunteered. Study population: 29 patients with unilateral cataract and contralateral pseudophakia. 


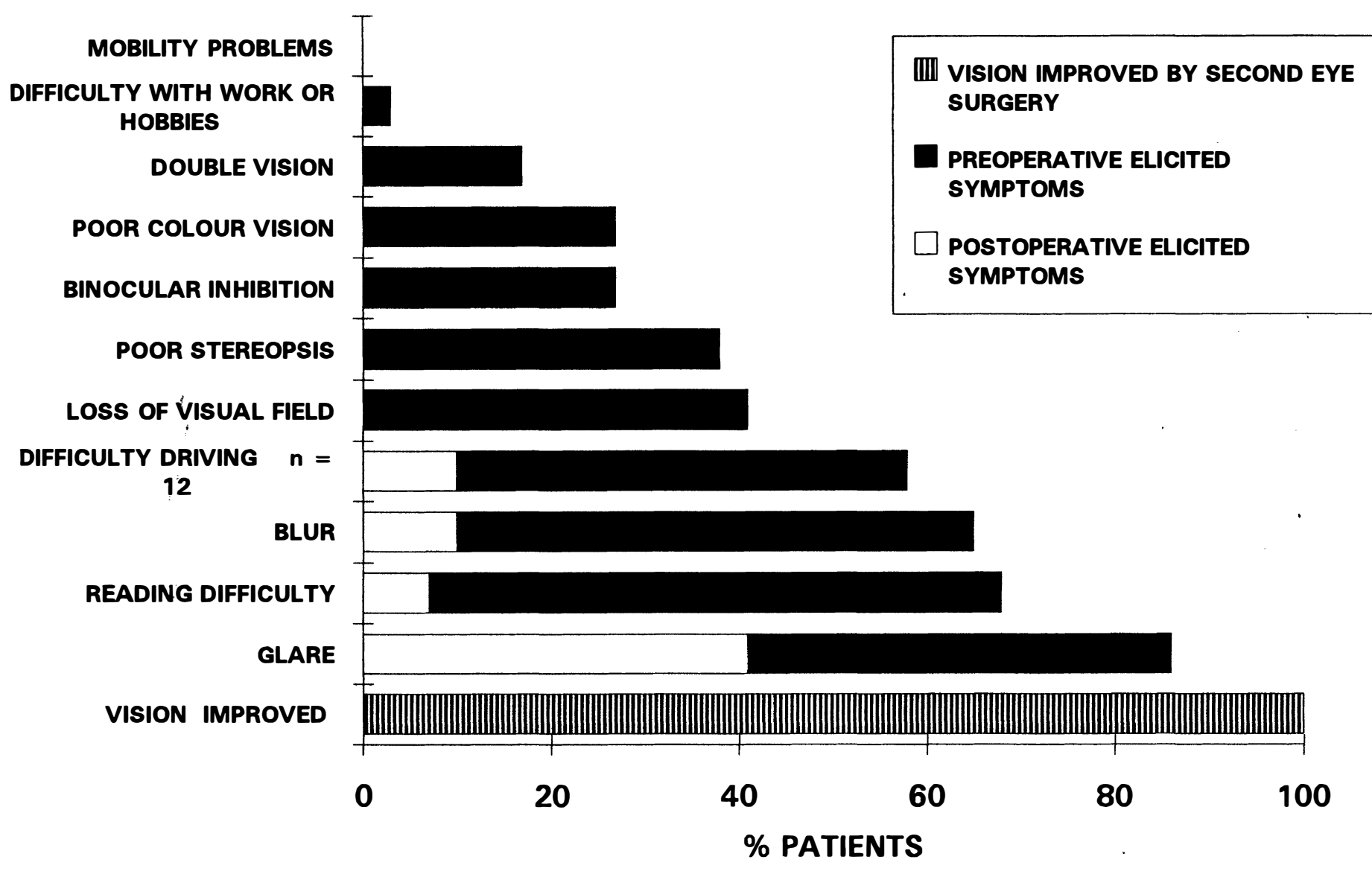

Fig. 2. Percentage of patients from whom each visual symptom was elicited by questionnaire before and after second eye cataract extraction. Percentage of patients reporting improved vision as a result of second eye surgery. Study population: 29 patients with unilateral cataract and contralateral pseudophakia.

There was no significant difference between the preoperative mean pseudophakic monocular visual acuity and pre-operative mean binocular visual acuity results $(p=0.74)$. Furthermore no individual demonstrated a difference between these two measurements of greater than four LogMAR letters (0.08 LogMAR).

Post-operatively the mean first and second eye acuities were 0.11 and $0.18 \operatorname{LogMAR}(6 / 7.7$ and 6/9.1). Following second eye surgery the mean binocular visual acuity was $0.063 \operatorname{LogMAR}(6 / 6.9)$. This is significantly better than the mean post-operative better eye visual acuity of 0.09 $\operatorname{LogMAR}(p=0.0082)$ and indicates the presence of binocular summation.

\section{Pelli-Robson Contrast Sensitivity}

Pre-operatively the mean pseudophakic eye PRCST was $1.44 \log$ units while that in the cataractous eye was 0.49 $\log$ units. There was no significant difference between the pre-operative mean monocular pseudophakic and preoperative mean binocular contrast sensitivity thresholds $(p=0.83)$. Additionally no individual showed a difference between these two measurements in excess of one triplet.

Following second eye surgery the mean better eye contrast sensitivity threshold was $1.61 \mathrm{log}$ units while that binocularly was $1.71 \log$ units. This difference is signifi- cant $(p=0.013)$ and indicates the presence of binocular summation. The mean operated eye contrast sensitivity threshold was improved to $1.51 \log$ units.

\section{Glare Disability}

The pre-operative pseudophakic eye mean glare disability was $0.18 \mathrm{log}$ units. The mean glare disability of cataractous eyes was $0.28 \mathrm{log}$ units. With a sample size of 29 patients this difference approaches but does not achieve significance $(p=0.084)$. This may suggest that an effect was present which our study was insufficiently powerful to detect. Following second eye cataract extraction the mean first and second eye glare disability was $0.24 \mathrm{log}$ units. No significant difference could be shown between the glare disability of pseudophakic eyes with and without posterior capsulotomies.

\section{DISCUSSION}

\section{Visual Symptoms}

When compared with similarly aged normal subjects the patients we studied had good uniocular pseudophakic and binocular visual acuities. Despite this we were able to elicit between two and eight different symptoms of visual disability from each patient (Figs. 1, 2). Symptoms of glare, difficulty reading and blurred vision were each elicited from over half the patients. All patients considered that 


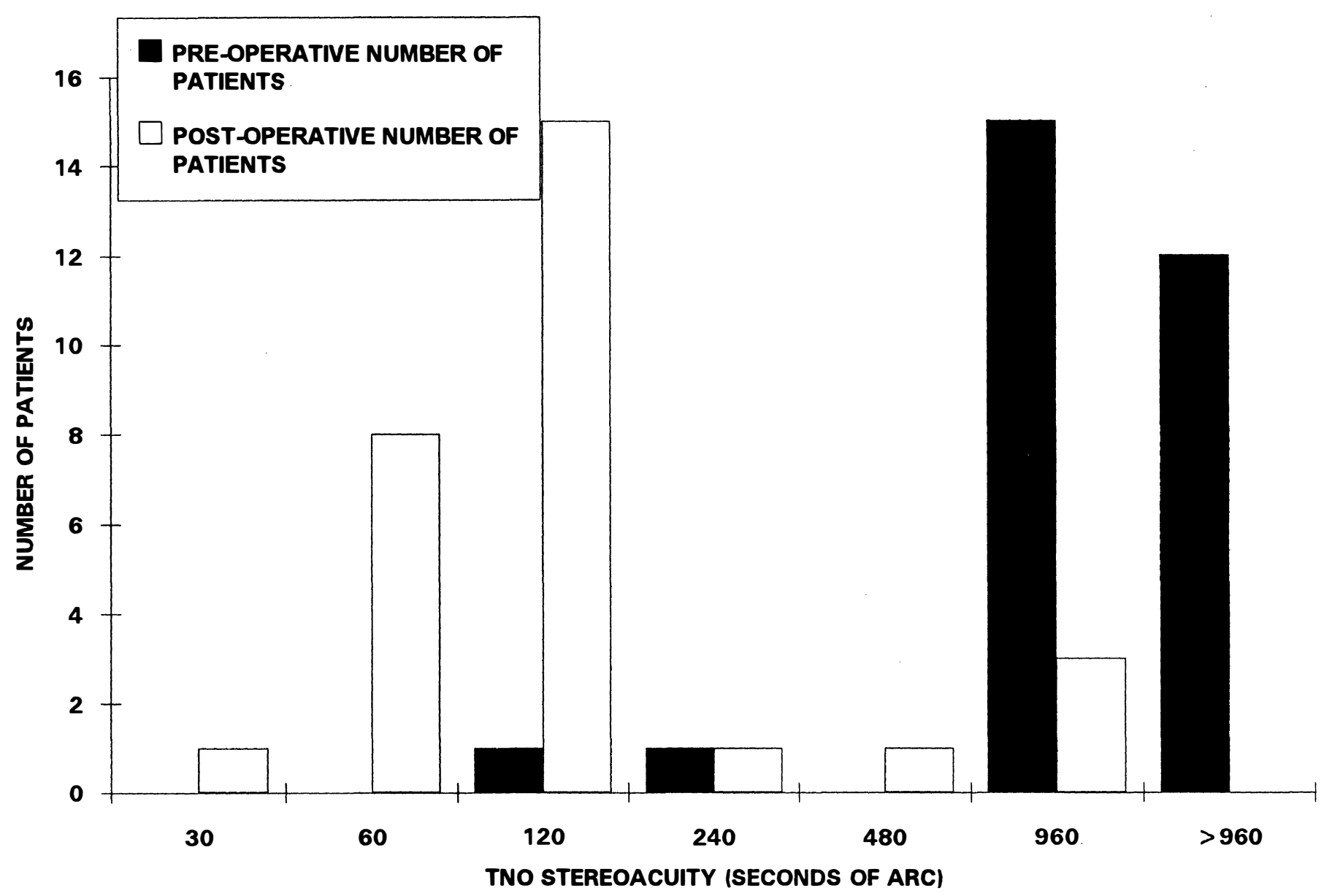

Fig. 3. TNO stereoacuity (seconds of arc) in 29 patients with unilateral cataract and contralateral pseudophakia before and after second eye cataract extraction.

their vision had been improved by second eye cataract extraction, the number of patients reporting each elicited symptom on direct questioning being dramatically reduced following second eye surgery. No patients volunteered any symptoms following second eye surgery.

The results of our observations suggest that symptomatically poor vision is the norm in patients with unilateral pseudophakia and contralateral cataract. Second eye cataract surgery greatly relieves these symptoms.

\section{Binocular Function}

Stereopsis was found to be better than 960 seconds of arc in only 2 pre-operative patients $(7 \%)$. Following surgery $86 \%$ could resolve a disparity of 120 seconds and $31 \%$ a disparity of 60 seconds or less (Fig. 3). A population study of 728 Londoners aged 65 years or over found stereo acuities exceeding approximately 340 seconds of arc in $71 \%$ of subjects and approximately 55 seconds of arc in $27 \%$ of subjects. ${ }^{20}$ Patients with unilateral cataract have poor stereopsis and frequent symptoms relating to judging distances. Second eye cataract extraction can restore normal stereopsis and relieves these symptoms.

The mean refractive anisometropia was reduced following second eye surgery. Whilst anisometropia inducing aniseikonia has been demonstrated to be a cause of poor stereopsis ${ }^{21}$ we did not demonstrate any correlation between post-operative anisometropia and stereo acuity. The improvement in stereo acuity would appear to be more dependent on improved high spatial frequency resolution than reduced refractive aniseikonia.

\section{Binocular Summation Following Second Eye Surgery}

Following second eye surgery the mean binocular visual acuity and contrast sensitivity thresholds in our group of patients were both significantly better than those from the best pseudophakic eye alone. Patients can therefore regain normal binocular summation of acuity and contrast sensitivity after bilateral cataract extraction. ${ }^{7,10,11,22,23}$

\section{Binocular Inhibition}

Prior to second eye surgery $28 \%$ of our subjects stated that they closed their cataractous eye in order to improve their vision (Fig. 1). Despite these symptoms we found no significant evidence of binocular inhibition. No individual demonstrated a binocular LogMAR acuity or Pelli-Robson contrast sensitivity which differed from that in their pseudophakic eye by more than the test-retest repeatability. Furthermore there was no significant difference between the mean pre-operative monocular and binocular visual acuity or contrast sensitivity threshold results.

Previous reports have suggested that binocular inhibi- 
tion occurs when the vision in each eye differs only slightly - the worse eye for example seeing $6 / 12$ or better. ${ }^{7-11}$ When there is a marked difference between the two eyes the input from the worse eye is ignored and binocular function then reflects that in the better eye alone. This was demonstrated in a patient with unilateral cataract and acuities of $6 / 5$ and $6 / 24$ in each eye respectively. ${ }^{8}$ This is called binocular suppression. We might therefore not expect patients with normal vision in a pseudophakic eye and anything other than early contralateral cataract to demonstrate binocular inhibition. Taylor et al. ${ }^{6}$ concluded that they had found evidence of binocular inhibition in over one third of a group of patients awaiting cataract surgery. They tested patients with Cambridge Low Contrast Gratings and LogMAR visual acuity charts. These patients had uniocular cataract inducing considerable differences in acuity or contrast threshold between the two eyes; the better eye was either normal or pseudophakic. Information on the individual interocular differences in acuity or contrast sensitivity was not presented and their results arise from single tests of acuity and contrast sensitivity. No allowance appears to have been made for possible test-retest variation when analysing the results and the level of acuity and contrast inhibition demonstrated exceeds that previously considered possible. ${ }^{7.10 .11}$ Patients with unilateral cataract frequently complain that their vision is improved if the cataractous eye is closed. The experimental models and available clinical data do not fully explain the basis of this troubling symptom and further work in this area is required.

\section{Glare}

It is not known whether symptoms of glare reflect the absolute level of contrast sensitivity threshold in bright light or the elevation in contrast threshold (glare disability) induced by bright light. Posterior chamber lens implants have previously been reported to be associated with glare. ${ }^{24}$ Eyes which underwent second eye cataract extraction showed a mean improvement in contrast sensitivity threshold of $1.02 \mathrm{log}$ units, approximately a tenfold increase. The mean glare disability of pseudophakic eyes following surgery was $0.24 \mathrm{log}$ units while that of the cataractous eyes was only slightly more at 0.28 log units. This difference is not significant $(p=0.67)$. The proportion of patients from whom symptomatic glare was elicited fell from $86 \%$ to $41 \%$ following second eye surgery. That the prevalence of symptomatic glare was not further reduced despite a tenfold increase in absolute contrast sensitivity threshold in the presence of glare suggests these symptoms may at least partly relate to the magnitude of glare disability.

There are no data on visual function in the presence of binocular glare and this may prove to be a more physiological test. Techniques for measuring binocular glare have not previously been described and need to be developed. ${ }^{17}$ The glare source must present an equal luminance to each eye, must not occlude one eye, and should not present a macular stress test to either eye.
We did not quantify symptom severity in this study and whilst glare symptoms persisted they may have been ameliorated. Further investigation is indicated in order to define the role of cataract extraction in reducing symptomatic glare.

\section{CONCLUSIONS}

We have shown that patients with normal vision in a pseudophakic eye but poor vision in a contralateral cataractous eye have multiple symptoms of poor vision. Symptoms suggesting that the cataractous eye is interfering with the function of the good eye are prevalent. Monocular and binocular contrast sensitivity threshold and visual acuity tests have, however, failed to demonstrate any significant evidence of binocular inhibition. Further investigation of binocular visual infection in patients with unilateral cataract is required in order to clarify the role of the second eye in inducing visual disability. Subjects with a unilateral cataract but contralateral pseudophakia retain binocular single vision but have poor stereopsis.

Second eye cataract extraction with lens implantation leads to a dramatic reduction in the proportion of patients reporting visual symptoms. Patients unanimously consider their vision to be improved by this procedure. Both normal stereopsis and binocular summation of visual acuity and contrast sensitivity may be regained following second eye cataract extraction.

Cataract surgery leads to a significant improvement in contrast sensitivity threshold both with and without glare, but the magnitude of glare disability is similar in cataractous and pseudophakic eyes. This may have some bearing on the persistence of symptomatic glare in some patients.

Patients with unilateral cataract and contralateral pseudophakia perceive their vision to be poor. Their symptomatic disability may be relieved by second eye cataract extraction and normal binocular function may be restored. Second eye cataract extraction has be shown to be beneficial.

The authors would like to acknowledge the help given by Joan Smith, Roger Ellingham and John Sparrow of the Bristol Eye Hospital and David Adams of the Department of Optometry, UWIST, Cardiff.

Key words: Binocularinhibition, Binocular summation, Binocular suppression, Cataract extraction, Glare, Stereopsis, Surgical outcome, Visual symptoms.

\section{REFERENCES}

1. Drummond MF. Economic aspects of cataract. Ophthalmology 1988;95:1147-53.

2. Bernth-Petersen P. Outcome of cataract surgery. IV. Socioeconomic aspects. Acta Ophthalmol (Copenh) 1982;60: 464-8.

3. Williams MH, Frankel SJ, Coast J, Nanchahal K. An epidemiologically based needs assessment: cataract surgery. Commissioned by NHS Management Executive. Bristol: Health Care Evaluation Unit, 1992.

4. Courtney P. The national cataract surgery survey. I. Method and descriptive features. Eye 1992;6:487-92.

5. The Oxford Cataract Treatment and Evaluation Team. Sub- 
jective assessment of the effects of cataract surgery and a review of long term aims. Eye 1987;1:247-53.

6. Taylor RH, Misson GP, Moseley MJ. Visual acuity and contrast sensitivity in cataracts: summation and inhibition of visual performance. Eye 1991;5:704-7.

7. Pardhan S, Gilchrist J. Binocular contrast sensitivity with monocular glare disability. Ophthalmic Physiol Opt 1990;10:37-9.

8. Pardhan S, Gilchrist J. The importance of measuring binocular contrast sensitivity in unilateral cataract. Eye 1991;5: 31-5.

9. Pardhan S. The measurement of binocular performance of unilateral cataract using the Regan test. Eye 1993;7:59-62.

10. Gilchrist J, Pardhan S, Binocular contrast detection with unequal monocular illuminance. Ophthalmic Physiol Opt 1987;7:373-7.

11. Pardhan S, Gilchrist J. The effect of monocular defocus on binocular contrast sensitivity. Ophthalmic Physiol Opt 1990;10:33-6.

12. Bennett AG, Rabbetts RB. Clinical vision optics. London: Butterworths, 1984: 276.

13. Elliot DB, Hearst MA, Weatherill J. Comparing clinical tests of visual function in cataract with the patient's perceived visual disability. Eye 1990;4:712-7.

14. Lovie-Kitchin JE. Validity and reliability of visual acuity measurements. Ophthalmic Physiol Opt 1988;8:363-70.

15. Pelli DG, Robson JG, Wilkins AJ. The design of a new letter chart for measuring contrast sensitivity. Clin Vis Sci 1988;2:187-99.

16. Elliot DB, Sanderson K, Conkey A. The reliability of the Pelli-Robson contrast sensitivity chart. Ophthalmic Physiol Opt 1990;10:21-4.

17. American Academy of Ophthalmology: Committee on Ophthalmic Procedures Assessment. Contrast sensitivity and glare testing in the evaluation of anterior segment disease. Ophthalmology 1990;97:1233-7.

18. Williamson TH, Strong NP, Sparrow J, Aggarwal RK, Harrad R. Contrast sensitivity and glare in cataract using the Pelli-Robson chart. Br J Ophthalmol 1992;76:719-22.

19. Paulsson LE, Sjöstrand J. Contrast sensitivity in the presence of a glare light: theoretical concepts and preliminary clinical studies. Invest Ophthalmol Vis Sci 1980;19:401-6.

20. Wright LA, Wormald RPL. Stereopsis and ageing. Eye 1992;6:473-6.

21. Katsumi O, Miyinaga Y, Hiroset et al. Binocular function in unilateral aphakia: correlation with aniseikonia and stereo acuity. Ophthalmology 1988;95:1088-93.

22. Heravian JS, Jenkins TCA, Douthwaite WA. Binocular summation, visually evoked responses and visual acuity. Ophthalmic Physiol Opt 1990;10:257-61.

23. Campbell F, Green D. Monocular versus binocular visual acuity. Nature 1965;208:191-2.

24. Nadler DJ, Jaffe NS, Clayman HM, et al. Glare disability in eyes with intraocular lenses. Am J Ophthalmol 1984; 97:43-7. 